recently come under the radar as a potential new biomarker for $\mathrm{HF}$ - one that is more cost-effective, less invasive, more convenient and acceptable for both patients and healthcare professionals, and one which could potentially reflect the information received from a standard blood NTproBNP test. One hurdle in measuring NTproBNP in saliva samples using standard point-of-care (POC) devices is the level of this protein in saliva. Measuring about 2000-fold lower, saliva NTproBNP is hardly measurable with standard POC devices. Here we present the KardiaTool platform, an integrated POC solution for non-invasive diagnosis and therapy monitoring of HF patients. The platform consists of two components, KardiaPOC (a highly sensitive portable device for non-invasive and simultaneous quantitative assessment of multiple HF related biomarkers from saliva samples, incorporating a functionalized magnetic nanoparticle approach ) and KardiaSoft (a decision support software based on predictive modelling techniques that analyses POC and other patient data, and delivers information on $\mathrm{HF}$ diagnosis and therapy monitoring). The KardiaTool platform was developed as part of an ongoing collaborative Horizon 2020 project, KARDIATOOL.

Methods and Results The aim of this sub-study was to test the hypothesis that saliva NTproBNP levels correlate to blood NTproBNP levels. To achieve this, we recruited chronic heart failure patients attending the HF unit in St. Michael's Hospital (SVUH, Dublin). Patients underwent routine physical examination, including venepuncture, echocardiography and electrocardiography. NTproBNP was quantified in peripheral blood samples. Saliva was collected with the SalivaBio Oral Swab device and extracted following centrifugation at $5000 \mathrm{~g}$ for 5 min. Saliva samples were assessed with a modified immunoassay prior to the official KardiaPOC testing. Results from the first $46 \mathrm{HF}$ patients show a significant and positive correlation between blood and saliva NTproBNP $(\mathrm{p}<0.05)$. In addition, saliva NTproBNP correlated significantly with echocardiography parameters, including LA diameter $(r=0.36, p=0.02)$ and E/A ratio $(r=0.43, p=0.01)$.

Conclusions We were successfully able to quantify saliva NTproBNP levels in HF patients using a modified immunoassay approach, and these levels correlated with blood levels. The KardiaTool project is still ongoing, with patient recruitment in Ireland, Greece, and Italy. The next stage is to test the KardiaPOC device, which we envisage will have superior sensitivity for saliva NTproBNP quantification, and to determine the diagnostic test accuracy for acute and chronic heart failure.

\section{NOVEL HEART FAILURE BIOMARKER CLEC3B IS ASSOCIATED WITH CARDIAC FIBROSIS, AND IMPACTS CARDIAC FIBROBLAST CELL FUNCTION IN VITRO}

${ }^{1} \mathrm{~K}$ Edgar, ${ }^{1} \mathrm{C}$ Tonry, ${ }^{2} \mathrm{~B}$ Collins, ${ }^{3} \mathrm{C}$ Moravec, ${ }^{4} \mathrm{M}$ Ledwidge, ${ }^{5} \mathrm{~K} \mathrm{McD}$ onald, ${ }^{6} \mathrm{P}$ Collier, ${ }^{1} \mathrm{D}$ Grieve, ${ }^{7} \mathrm{C}$ Watson. ${ }^{1}$ Wellcome-Wolfson Institute for Experimental Medicine, Queens University Belfast, UK; ${ }^{2}$ School of Biological Sciences, Queen's University Belfast, UK; ${ }^{3}$ Department of Cardiovascular and Metabolic Sciences, Lerner Research Institute, Cleveland Clinic, Ohio, USA; ${ }^{4}$ Heartbeat Trust, Dublin, Ireland; ${ }^{5}$ St. Vincent's University Hospital, Dublin, Ireland; ${ }^{6}$ Cleveland Clinic, USA; ${ }^{7}$ Queen's University Belfast, UK

10.1136/heartjnl-2021-ICS.41

Background Through a proteomics approach we have recently identified a novel blood-based biomarker for heart failure called CLEC3B, which exhibits improved diagnostic accuracy over BNP. We have previously investigated CLEC3B expression in human atrial tissue and shown that it correlates positively with the degree of cardiac tissue fibrosis. The purpose of this study was to expand our current knowledge of CLEC3B in the context of heart failure, with a specific emphasis on cardiac fibrosis.

Methods Left ventricular cardiac tissue samples from 30 patients with heart failure and 9 age/sex matched non-heart failure controls were studied to assess protein levels of CLEC3B and markers of cardiac fibrosis. As the primary cell type contributing to cardiac fibrosis is the fibroblast, parallel in vitro studies were carried out using primary human cardiac fibroblast cells (HCF). Both CLEC3B gene over-expression and gene knock-down studies were carried out in HCFs, in the presence or absence of stimulation with the pro-fibrotic growth factor TGF $\beta$. The impact of modulating endogenous CLEC3B levels on gene and protein expression was studied, including by RNA sequencing, to identify the potential role of CLEC3B in fibroblast cells. An in vivo model of Angiotensin II induced heart failure in CLEC3B knock out mice is currently ongoing to study the impact of CLEC3B on cardiac function and remodelling (echocardiography, histology, gene and protein analysis).

Results Protein levels of CLEC3B were significantly increased in cardiac tissue derived from patients with heart failure $(p<0.01)$, including in both $\operatorname{HFrEF}(p<0.001)$ and HFpEF $(p<0.01)$ sub-sets, compared to non-failure controls. Levels of CLEC3B in cardiac tissue correlated significantly with markers of fibrosis, including collagen subtypes 1, 5, 6,12 , and 14 , with the r-statistic ranging between 0.31 and 0.78, (all p<0.05). RNAseq analysis of HCF cells showed significant changes in over 400 genes following both CLEC3B over-expression and knockdown, with different genes being modulated depending on TGF $\beta$ stimulation of the HCF cells.

Conclusions/Implications These data show how CLEC3B has an important role in the fibrosis associated with cardiac remodelling in heart failure. Many candidate genes have been identified from RNAseq analysis which will help guide future investigations, alongside the in vivo CLEC3B knockout animal model of heart failure, to help elucidate the mechanisms involved in the role of CLEC3B in cardiac remodelling and disease progression.

\section{RENAL SAFETY OF SGLT2 INHIBITORS IN THE REAL WORLD - AN OUTPATIENT HEART FAILURE CLINIC EXPERIENCE}

R O'Grady, BF McAdam, L O'Connor, M Kelly. Beaumont Hospital, Dublin, Ireland

\subsection{6/heartjnl-2021-ICS.42}

Background As recent evidence has emerged indicating the efficacy of sodium-glucose-co-transporter-2 inhibitors (SGLT2i) in reducing all-cause mortality as well as hospitalisations in patients with HFrEF, it has been widely and quickly adapted into Cardiology practice. However, given the comorbidities and polypharmacy associated with this patient cohort, the real-world initiation and monitoring can be challenging.

Aim To assess the renal biochemical effects of SGLT2i initiation, as well as effects on hospitalisation and side effects in a 